\title{
A new approach for separating mixed model parameters: application to simultaneous inversion of earthquake source parameters
}

\author{
Weijian Mao
}

Received: 7 August 2013/ Accepted: 14 November 2013/Published online: 10 December 2013

(C) The Seismological Society of China, Institute of Geophysics, China Earthquake Administration and Springer-Verlag Berlin Heidelberg 2013

\begin{abstract}
A method for simultaneous determination of mixed model parameters, which have different physical dimensions or different responses to data, is presented. Mixed parameter estimation from observed data within a single model space shows instabilities and trade-offs of the solutions. We separate the model space into $N$-subspaces based on their physical properties or computational convenience and solve the $N$-subspaces systems by damped least-squares and singular-value decomposition. Since the condition number of each subsystem is smaller than that of the single global system, the approach can greatly increase the stability of the inversion. We also introduce different damping factors into the subsystems to reduce the tradeoffs between the different parameters. The damping factors depend on the conditioning of the subsystems and may be adequately chosen in a range from $0.1 \%$ to $10 \%$ of the largest singular value. We illustrate the method with an example of simultaneous determination of source history, source geometry, and hypocentral location from regional seismograms, although it is applicable to any geophysical inversion.
\end{abstract}

Keywords Separation of model parameters . Damped least-squares · Singular value decomposition (SVD) · Source inversion

\section{W. Mao $(\square)$}

Center for Computational and Exploration Geophysics, Institute of Geodesy and Geophysics, Chinese Academy of Sciences, Wuhan 430077, China

e-mail:wjmao@whigg.ac.cn

W. Mao

State Key Laboratory of Geodesy and Earth's Dynamics, Institute of Geodesy and Geophysics, Chinese Academy of Sciences, Wuhan 430077, China

\section{Introduction}

Geophysical inversion may involve the estimation of various model parameters from a set of observed data. For example, in matter of earthquake source inversion at a local or regional scale, it is often possible and desirable, to invert at once for the source mechanism, the source location and the source history. However, because responses of data to different model parameters are different, the elements of the coefficient matrix have different orders of magnitude, which generally lead to ill conditioned and inhomogeneously solved matrices. In order to overcome this problem, a weighting matrix can be introduced to balance the large difference between the elements of the coefficient matrix (Mao et al. 1994). The weighting matrix method can stabilize the inverted solution, but sometimes also leads to spurious results (see Sect. 2 for details). And this method is mostly valid to correct for magnitude discrepancies, but does not take into account possible trade-offs in the determination of the different parameters.

In the present problem, we deal with three different classes of physical parameters, and solving for all of them on an equal basis would probably give priority to particular ones and bias estimates of the others. We also deal with non-linear inverse problems, since only the resolution of a moment tensor or of the source time function might be simple linear problems. A general approach to solve a reduced set of parameters in a non-linear way is to use gradient methods. Such a method for the inversion of the hypocentral relocation, of a double couple, and of the source time function, has been developed by Mao et al. (1994) and applied to local events by Nformi et al. (1996). In order to take into account the discrepancies between the three classes of physical parameters, the use of a weighting matrix had been proposed by Mao et al. (1994). 
We propose here a new approach based on the concept of separation of parameters. Such an approach has already been proposed by several authors for problems usually related to the inversion of structural models. A classical one is the 2-subsystems $(2 S)$ simultaneous inversion for hypocentral location and velocity structure. For example, Pavlis and Booker (1980) solved this problem in terms of a mixed discrete-continuous problem. Spencer and Gubbins (1980), followed by Mao and Suhadolc (1992), use partitioned matrices. Mao and Gubbins (1995) applied the method to seismic beam forming, and Mao and Stuart (1997) to multiphase seismic tomography. More basic references are given by Spencer (1985). Kennett et al. (1988) also proposed a method of minimization in local subspaces, applied to various problems of structural seismology.

In this paper, we use the damped least-squares technique and the singular value decomposition (SVD) for the simultaneous inversion of NS. We first expose the separation of parameters in two classes, following the mathematical basis of Mao and Suhadolc (1992) and Mao and Stuart (1997). Then we extend the previous idea to the separation of the three classes $(3 S)$ of source parameters, and finally derive the formulation of the $N S$ approach. Some comparisons on synthetic tests between the $2 S$ and $3 S$ approaches for the determination of source parameters are given. An application of the $3 S$ method to a real case, the regional study of the $M_{\mathrm{W}}=5.4$ Roermond earthquake (The Netherlands, April 13, 1992; Dufumier et al. 1997) is presented.

\section{General presentations}

We use a gradient method for the iterative determination of the parameters of a double couple (strike, dip, and rake angles), of the source time function (parameterized by $M$ overlapping triangles) and of the hypocenter location (longitude, latitude, and depth). The method is described by Mao et al. (1994) and solves iteratively the following linearized normal system.

$\boldsymbol{G}^{\mathrm{T}} \boldsymbol{G} \delta \boldsymbol{p}=\boldsymbol{G}^{\mathrm{T}} \boldsymbol{r}$,

where $T$ denotes the transposed matrix, $\delta \boldsymbol{p}$ is the perturbation vector to be added at each step to the $M+6$ model parameters $\boldsymbol{p}, \boldsymbol{r}$ is the residual vector between the observed data and the synthetics, and $\boldsymbol{G}$ is the corresponding matrix of the derivatives of Frechet.

The system is solved by singular value decomposition:

$$
\delta \boldsymbol{p}=\left(\boldsymbol{G}^{\mathrm{T}} \boldsymbol{G}\right)^{-1} \boldsymbol{G}^{\mathrm{T}} \boldsymbol{r}=\boldsymbol{V} \boldsymbol{S}^{-1} \boldsymbol{U}^{\mathrm{T}} \boldsymbol{r}
$$

where $\boldsymbol{V}$ and $\boldsymbol{U}$ are orthogonal matrices, $\boldsymbol{S}$ is a diagonal matrix formed by the singular values of the matrix $\boldsymbol{G}$.
A damping factor $\alpha$ is introduced to eliminate the risks of instability due to the possible presence of very small singular values of $\boldsymbol{G}$ :

$\delta \boldsymbol{p}=\boldsymbol{G}^{+} \boldsymbol{r}$

where $\boldsymbol{G}^{+}=\left(\boldsymbol{G}^{\mathrm{T}} \boldsymbol{G}+\alpha \boldsymbol{I}\right)^{-1} \boldsymbol{G}^{\mathrm{T}}=\boldsymbol{V}\left(\boldsymbol{S}^{2}+\alpha \boldsymbol{I}\right)^{-1} \boldsymbol{S} \boldsymbol{U}^{\mathrm{T}}$ is the generalized inverse of $\boldsymbol{G}$, and $\boldsymbol{I}$ is the identity matrix.

In order to take into account the large discrepancies between the various physical dimensions of the elements of the matrix $\boldsymbol{G}$, Mao et al. (1994) introduced a weighting matrix $\boldsymbol{W}$, solving the transformed system for the "weighted" parameters $\delta \mathbf{p}^{\prime}$ :

$\boldsymbol{G W} \delta \boldsymbol{p}^{\prime}=\boldsymbol{r}$

where $\boldsymbol{W}$ is allowed to be a diagonal or off-diagonal matrix. The weighting matrix method may be a simple suitable way to correct for the different orders of magnitude of the elements of the coefficient matrix $\boldsymbol{G}$, but it may also lead sometimes to spurious results. For example, $\boldsymbol{W}$ may contain some elements of very large value in order to balance very small elements of $\boldsymbol{G}$. These large elements of $\boldsymbol{W}$ affect adversely the final model $\delta \boldsymbol{p}=\boldsymbol{W} \delta \boldsymbol{p}^{\prime}$ (the error in $\delta \boldsymbol{p}^{\prime}$ is spuriously enlarged by $\boldsymbol{W}$, even if initially small). In addition, the full matrix inversion looses computational efficiency.

We describe here an alternative approach of the same problem, replacing the method of the "weighting matrix" by the separation of a mixed parameter space into several subspaces.

\section{The 2-subsystems (2S)}

We first present the separation of parameters in two classes, following the mathematical basis of Mao and Suhadolc (1992) for the resolution of the hypocenter location and of the velocity structure, and of Mao and Stuart (1997) for the location of interfaces and the distribution of velocities. Our first group of parameters, $\boldsymbol{p}_{1}$, is the hypocentral location, while the second group, $\boldsymbol{p}_{2}$, is composed of the source mechanism and of the time function. We will therefore now use the notation $\boldsymbol{p}=\left(\boldsymbol{p}_{1}\right.$, $\left.\boldsymbol{p}_{2}\right)$, and $\boldsymbol{G}=\left(\boldsymbol{G}_{1}, \boldsymbol{G}_{2}\right)$.

The linearized system of damped normal equations becomes:

$$
\begin{aligned}
& \left(\boldsymbol{O}_{1} \boldsymbol{G}_{1}+\alpha_{1} \boldsymbol{I}\right) \delta \boldsymbol{p}_{1}=\boldsymbol{O}_{1} \boldsymbol{r}, \\
& \left(\boldsymbol{G}_{2}^{\mathrm{T}} \boldsymbol{G}_{2}+\alpha_{2} \boldsymbol{I}\right) \delta \boldsymbol{p}_{2}=\boldsymbol{G}_{2}^{\mathrm{T}}\left(\boldsymbol{r}-\boldsymbol{G}_{1} \delta \boldsymbol{p}_{1}\right),
\end{aligned}
$$

where $\boldsymbol{O}_{1}=\boldsymbol{G}_{1}^{\mathrm{T}}-\boldsymbol{G}_{1}^{\mathrm{T}} \boldsymbol{G}_{2} \boldsymbol{G}_{2}^{+}$, and $\alpha_{1}, \alpha_{2}$ are the damping factors.

Each system is solved by singular value decomposition. The solution of Eq (5) is 


$$
\begin{array}{r}
\delta \boldsymbol{p}_{1}=\left(\boldsymbol{O}_{1} \boldsymbol{G}_{1}\right)^{+} \boldsymbol{O}_{1} \boldsymbol{r}, \\
\delta \boldsymbol{p}_{2}=\boldsymbol{G}_{2}^{+}\left(\boldsymbol{r}-\boldsymbol{G}_{1} \delta \boldsymbol{p}_{1}\right),
\end{array}
$$

where + denotes the generalized inverse, defined in Eq (3).

\section{The 3-subsystems (3S)}

We now extend the formulation of the separation of parameters to three different classes: mechanism, source time function, and hypocentral location. The choice seems natural not only because these groups are associated to physically different quantities, but also because the parameters inside each class are now of the same type, measured with the same unit, and of the same order of magnitude (note that for the epicenter, we do not invert directly for the latitude and the longitude, but for epicentral shifts in kilometers, which are directly comparable to the shift in depth).

We can now write $\boldsymbol{p}=\left(\boldsymbol{p}_{1}, \boldsymbol{p}_{2}, \boldsymbol{p}_{3}\right)$, and $\boldsymbol{G}=\left(\boldsymbol{G}_{1}, \boldsymbol{G}_{2}\right.$, $\left.\boldsymbol{G}_{3}\right)$, and the damped normal system becomes:

$$
\begin{aligned}
& \left(\boldsymbol{O}_{1} \boldsymbol{G}_{1}+\alpha_{1} \boldsymbol{I}\right) \delta \boldsymbol{p}_{1}=\boldsymbol{O}_{1} \boldsymbol{r} \\
& \left(\boldsymbol{O}_{2} \boldsymbol{G}_{2}+\alpha_{2} \boldsymbol{I}\right) \delta \boldsymbol{p}_{2}=\boldsymbol{O}_{2}\left(\boldsymbol{r}-\boldsymbol{G}_{1} \delta \boldsymbol{p}_{1}\right) \\
& \left(\boldsymbol{G}_{3}^{\mathrm{T}} \boldsymbol{G}_{3}+\alpha_{3} \boldsymbol{I}\right) \delta \boldsymbol{p}_{3}=\boldsymbol{G}_{3}^{\mathrm{T}}\left(\boldsymbol{r}-\boldsymbol{G}_{1} \delta \boldsymbol{p}_{1}-\boldsymbol{G}_{2} \delta \boldsymbol{p}_{2}\right) .
\end{aligned}
$$

The solution vectors are given by

$$
\begin{aligned}
& \delta \boldsymbol{p}_{1}=\left(\boldsymbol{O}_{1} \boldsymbol{G}_{1}\right)^{+} \boldsymbol{O}_{1} \boldsymbol{r}, \\
& \delta \boldsymbol{p}_{2}=\left(\boldsymbol{O}_{2} \boldsymbol{G}_{2}\right)^{+} \boldsymbol{O}_{2}\left(\boldsymbol{r}-\boldsymbol{G}_{1} \delta \boldsymbol{p}_{1}\right), \\
& \delta \boldsymbol{p}_{3}=\boldsymbol{G}_{3}^{+}\left(\boldsymbol{r}-\boldsymbol{G}_{1} \delta \boldsymbol{p}_{1}-\boldsymbol{G}_{2} \delta \boldsymbol{p}_{2}\right),
\end{aligned}
$$

where

$$
\begin{aligned}
& \boldsymbol{O}_{1}=\boldsymbol{G}_{1}^{\mathrm{T}} \boldsymbol{Q}_{3} \boldsymbol{Q}_{2}, \\
& \boldsymbol{O}_{2}=\boldsymbol{G}_{2}^{\mathrm{T}} \boldsymbol{Q}_{3}, \\
& \boldsymbol{Q}_{2}=\boldsymbol{I}-\boldsymbol{G}_{2}\left(\boldsymbol{O}_{2} \boldsymbol{G}_{2}\right)^{+} \boldsymbol{O}_{2}, \\
& \boldsymbol{Q}_{3}=\boldsymbol{I}-\boldsymbol{G}_{3} \boldsymbol{G}_{3}^{+} .
\end{aligned}
$$

In order to optimize the mixed parameter inversion process, it is important to invert first the most stable subsystem. We have chosen in our application to invert first the hypocentral location, which is supposed to be the best known quantity a priori, and which, from our experience, presents the best conditioning among the $3 S$. We then invert the mechanism, and finally the source time function which is the most unstable component of the problem.

\section{The $N$-subsystems (NS)}

We extend the $3 S$ formulation to the $N S$ case. Assuming $\boldsymbol{p}=\left(\boldsymbol{p}_{1}, \boldsymbol{p}_{2}, \ldots, \boldsymbol{p}_{N}\right)$, and $\boldsymbol{G}=\left(\boldsymbol{G}_{1}, \boldsymbol{G}_{2}, \ldots, \boldsymbol{G}_{N}\right)$, we have the corresponding formulations:

$$
\begin{aligned}
& \delta \boldsymbol{p}_{1}=\left(\boldsymbol{O}_{1} \boldsymbol{G}_{1}\right)^{+} \boldsymbol{O}_{1} \boldsymbol{r} \\
& \delta \boldsymbol{p}_{2}=\left(\boldsymbol{O}_{2} \boldsymbol{G}_{2}\right)^{+} \boldsymbol{O}_{2}\left(\boldsymbol{r}-\boldsymbol{G}_{1} \delta \boldsymbol{p}_{1}\right), \\
& \delta \boldsymbol{p}_{\mathrm{N}-1}=\left(\boldsymbol{O}_{\mathrm{N}-1} \boldsymbol{G}_{\mathrm{N}-1}\right)^{+} \boldsymbol{O}_{\mathrm{N}-1}\left(\boldsymbol{r}-\sum_{\mathrm{i}=1}^{N-2} \boldsymbol{G}_{i} \delta \boldsymbol{p}_{i}\right) \\
& \delta \boldsymbol{p}_{\mathrm{N}}=\boldsymbol{G}_{\mathrm{N}}^{+}\left(\boldsymbol{r}-\sum_{\mathrm{i}=1}^{\mathrm{N}-1} \boldsymbol{G}_{\mathrm{i}} \delta \boldsymbol{p}_{\mathrm{i}}\right)
\end{aligned}
$$

where

$$
\begin{aligned}
& \boldsymbol{O}_{1}=\boldsymbol{G}_{1}^{\mathrm{T}} \boldsymbol{Q}_{\mathrm{N}} \boldsymbol{Q}_{\mathrm{N}-1} \ldots \boldsymbol{Q}_{3} \boldsymbol{Q}_{2}, \\
& \boldsymbol{O}_{2}=\boldsymbol{G}_{2}^{\mathrm{T}} \boldsymbol{Q}_{\mathrm{N}} \boldsymbol{Q}_{\mathrm{N}-1} \ldots \boldsymbol{Q}_{3}, \\
& \boldsymbol{O}_{\mathrm{N}-1}=\boldsymbol{G}_{\mathrm{N}-1}^{\mathrm{T}} \boldsymbol{Q}_{\mathrm{N}},
\end{aligned}
$$

and

$$
\begin{aligned}
& \boldsymbol{Q}_{2}=\boldsymbol{I}-\boldsymbol{G}_{2}\left(\boldsymbol{O}_{2} \boldsymbol{G}_{2}\right)^{+} \boldsymbol{O}_{2}, \\
& \boldsymbol{Q}_{3}=\boldsymbol{I}-\boldsymbol{G}_{3}\left(\boldsymbol{O}_{3} \boldsymbol{G}_{3}\right)^{+} \boldsymbol{O}_{3}, \\
& \boldsymbol{Q}_{\mathrm{N}-1}=\boldsymbol{I}-\boldsymbol{G}_{\mathrm{N}-1}\left(\boldsymbol{O}_{\mathrm{N}-1} \boldsymbol{G}_{\mathrm{N}-1}\right)^{+} \boldsymbol{O}_{\mathrm{N}-1}, \\
& \boldsymbol{Q}_{\mathrm{N}}=\boldsymbol{I}-\boldsymbol{G}_{\mathrm{N}} \boldsymbol{G}_{\mathrm{N}}^{+} .
\end{aligned}
$$

It should be noted that the method of parameter separation does not neglect the possible trade-offs and couplings between the different parameters, which are and must be taken into account in the inverse problem. But we can reduce the trade-offs by using different damping factors which are dependent on the conditioning of the subsystems. In general, the damping factor should be chosen in a range from $0.1 \%$ to $10 \%$ of the largest singular value. It is also possible, using different damping factors, to control the convergence of the inversion. For example, a relatively heavy damping can be given to a priori well-known parameters, so that the minimum of the misfit function is obtained by mostly varying the other model parameters.

\section{Comparison of the 2-and 3-subsystems methods}

The immediate advantage of the separation of parameters is the possibility to follow and control separately each part of the inversion for the source model. In particular, the condition numbers (e.g., Tarantola 1987) of each problem can be examined a priori, independently from the data, and three different damping factors can be used appropriately to stabilize the systems which may appear to be ill conditioned.

We show in Fig. 1 the condition numbers associated with the $3 S$ (mechanism, source time function, location) and with $2 S$ only (mechanism + source time function, and location) for different station distributions. The data are the vertical components of waveforms at regional distances (200 to $800 \mathrm{~km}$ ), at periods of $5 \mathrm{~s}$ and more. The 6-stations distribution offers a full azimuthal coverage, and then we 
System Stability as a function of the number of Stations

for the 2 and 3 subsystems methods
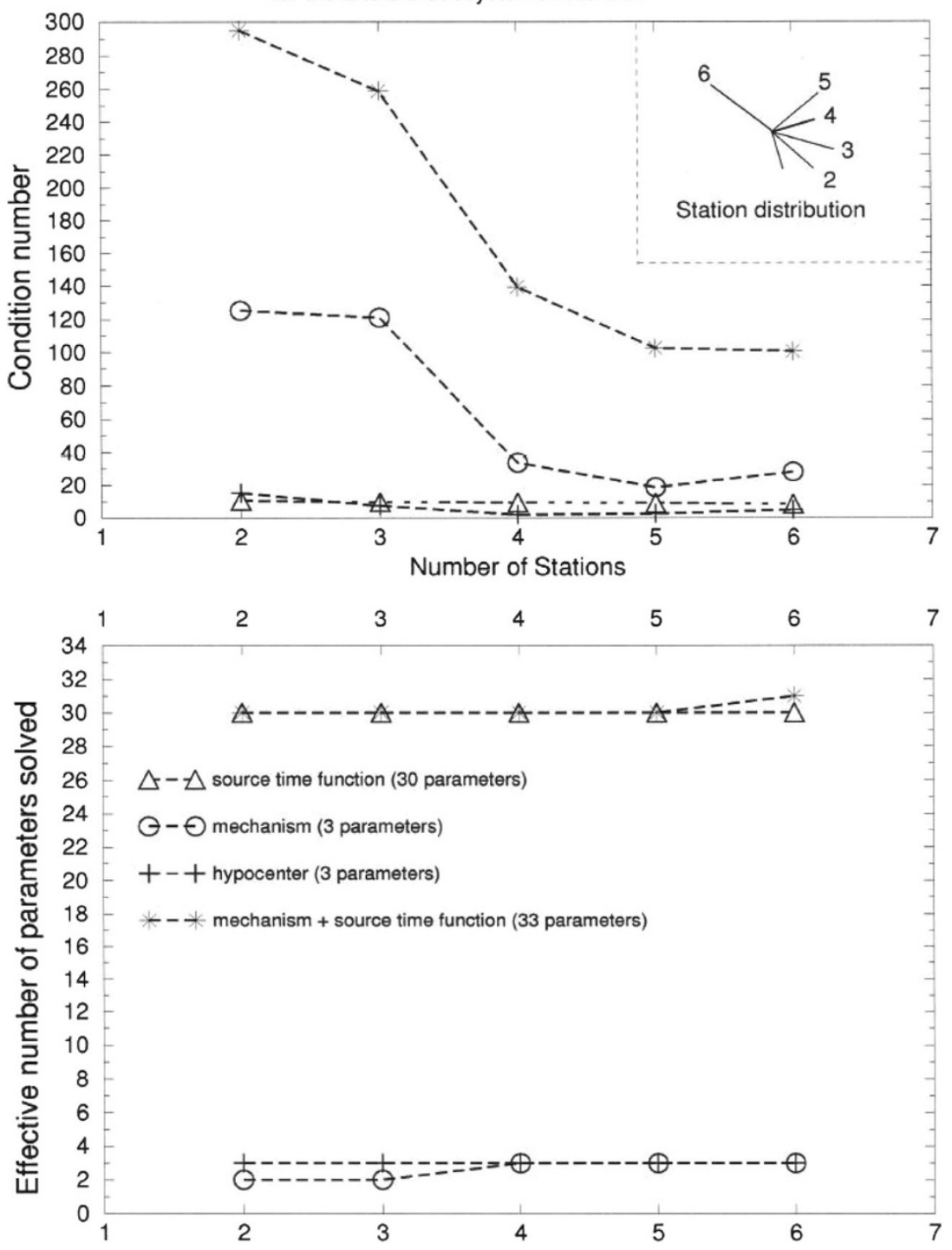

Fig. 1 Top variation of the condition number for the $4 S$ solving the source time function (triangles), the mechanism (circles), the hypocenter (crosses), and the source time function and the mechanism together (stars). We present the results as a function of the number of stations, starting from the 6-stations distribution shown in the upper right corner and then eliminating successively the stations number 6, 5, 4, and 3. Bottom Effective number of parameters solved in each subsystem, using a damping ratio of 0.001 for the same subsystems. The mechanism is described by 3 parameters, which appear not to be correctly solved when using less than 4 stations, i.e., an azimuthal aperture less than $80^{\circ}$. The source time function is parameterized here by 30 overlapping triangles, so that the (mechanism + source time function) system should solve for 33 parameters

progressively reduce the number of stations diminishing the azimuthal aperture. The subsystems including the mechanism as unknown appear to be unstable when less than 4 stations are used, i.e., when the total azimuthal aperture is less than $80^{\circ}$. This is confirmed below by the fact that only 2 of the 3 parameters of a double-couple can then be found when using a relatively small damping ratio of 0.001 (i.e., a damping factor equal to $0.1 \%$ of the largest singular value).

We remark that this undetermination of the mechanism is reported in the (mechanism + source time function) subsystem of the $2 S$ problem. Therefore it may affect also the resolution of the source time function. Indeed, the number of parameters solved inside the common subsystem is only of 30 or 31 parameters out of 33 (30 triangles in the parameterization of the time function plus 3 parameters for the mechanism), for a damping ratio of 0.001 . While using the complete separation of parameters, all the 30 parameters of the time function are solved, and the total proportion of parameters solved is of 32 or 33 out of 33 .

We also performed some synthetic tests with the $2 S$ and $3 S$ methods in order to test the improvements brought 


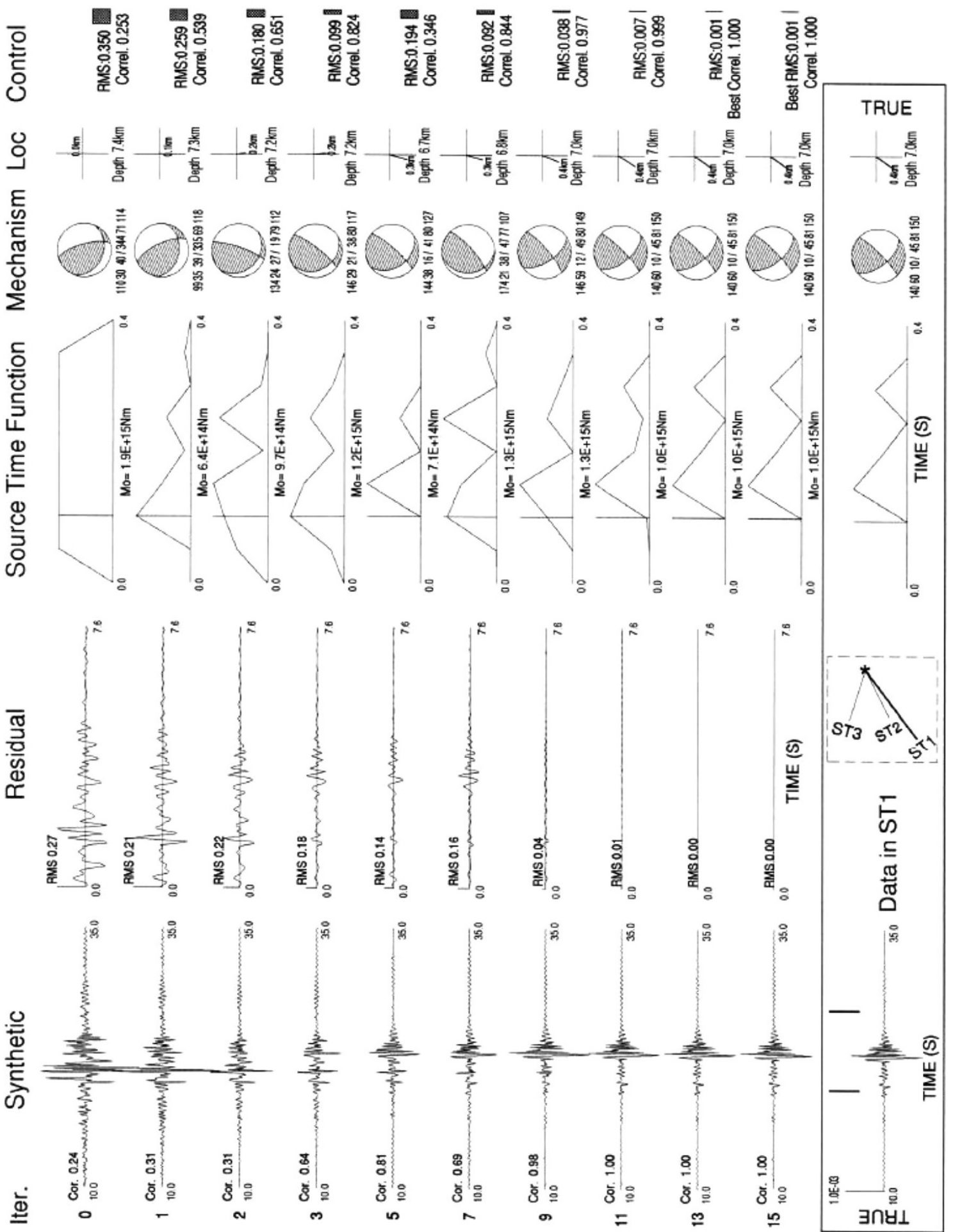

Fig. 2 Evolution of the source model using the $3 S$ approach for the selection of iterations. Iteration 0 corresponds to the initial source parameters and corresponding data. The true model and data are shown in the lower solid box. The very poor, station distribution used is shown in the dashed box. At each iteration, are shown from left to right: the waveform and residuals of the station ST1, the source time function (the vertical bar indicates the true origin time), the mechanism, the hypocentral shift (the cross indicates the $1 \mathrm{~km}$ scale), the average RMS and the normalized correlation coefficient. The inversion converges to the exact model in 15 iterations 


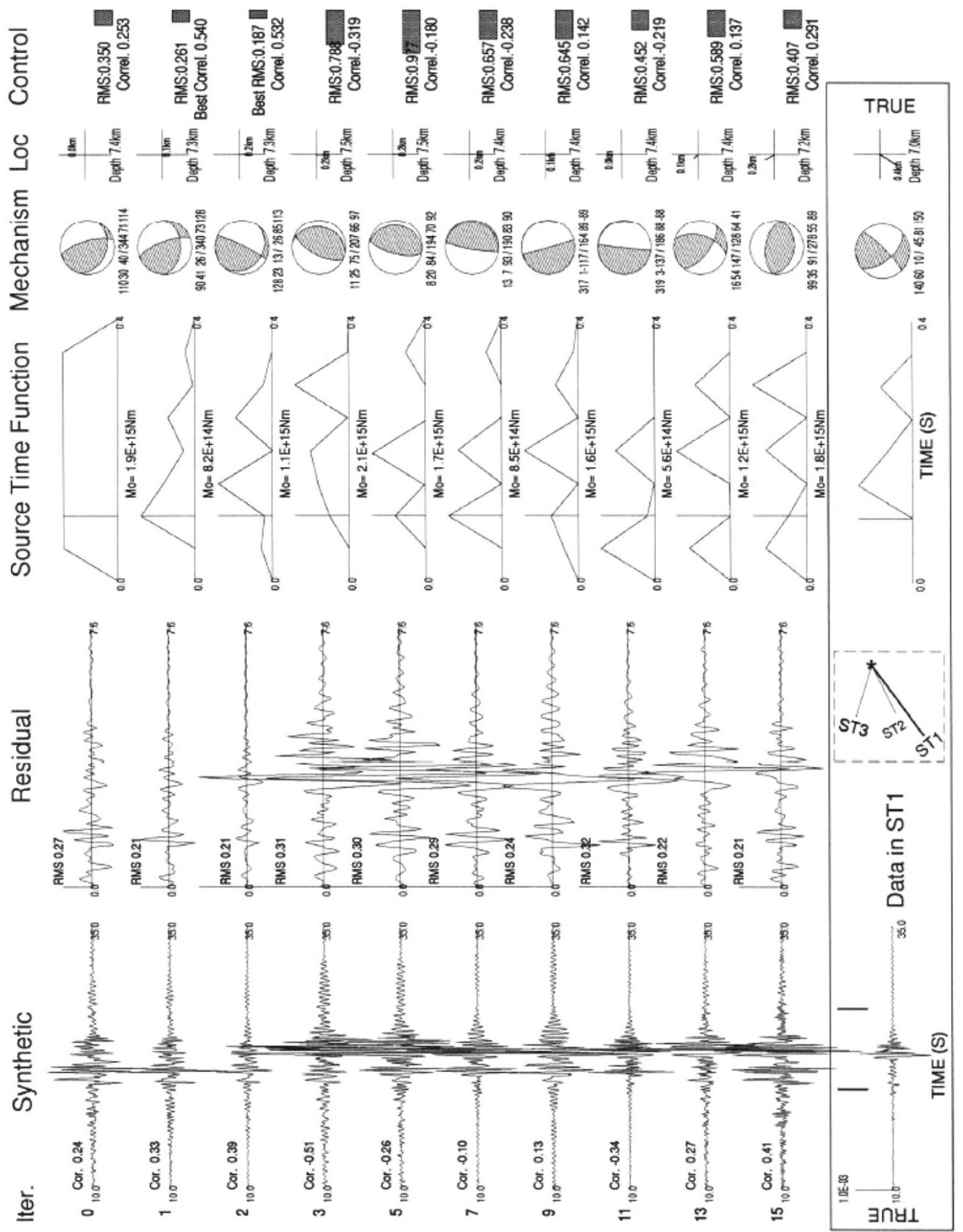

Fig. 3 Evolution of the source model using the $2 S$ approach for the selection of iterations.It shows the diverging behavior of the $2 S$ method. The best fit is obtained at the second iteration, and is similar to the $3 \mathrm{~S}$ model at the same level, but the result remains poor in the further iterations

about the separation of the mechanism and of the source time function in some critical cases. It is clear that, in presence of non-perturbated synthetic data and starting from an a priori model close enough to the "true" one to be inside the global minimum of the misfit function, both methods converge to the exact solution. But when one of the physical quantities is intrinsically poorly determined (for example the mechanism when the station distribution offers a poor aperture), or when some instability of the model may perturb the iterative process (for example when 


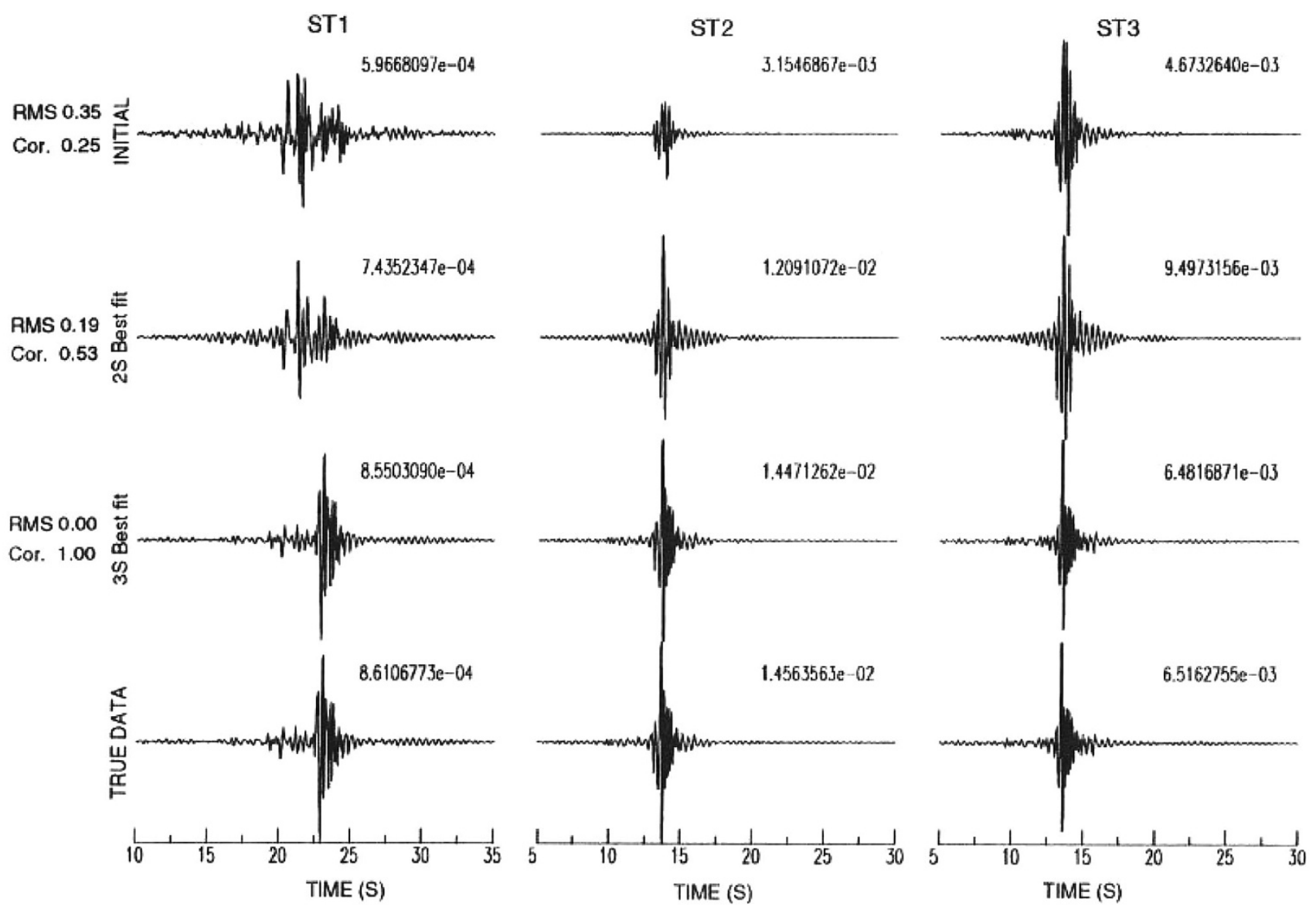

Fig. 4 Waveform fitting between the true data (bottom), the initial ones, and the $2 S$ and $3 S$ best-fitting ones, for all of the three stations used in the inversion. The number above each seismogram is its maximum amplitude. The average RMS error and normalized correlation coefficients (Cor) are indicated on the left

the complexity of the source time function cannot be fully solved from the frequency content of the date), the two methods may follow different convergence paths.

We show in Figs. 2 and 3 such a comparative examples. We built synthetic waveforms up to $10 \mathrm{~Hz}$ for three relatively close stations (the station distribution is symbolized at the bottom of the figures), at epicentral distances of $40-70 \mathrm{~km}$. The true model is a mechanism with a strike of $140^{\circ}$, a dip of $60^{\circ}$ and a rake angle of $10^{\circ}$, the source time function shown in the lower right corner, at a depth of $7 \mathrm{~km}$. The initial starting model is located $400 \mathrm{~m}$ deeper and $400 \mathrm{~m}$ more to the North-East, with a much longer source time function and $30^{\circ}$ of error on each angle of the mechanism. Note that the data corresponding to this initial model (iteration 0 ) strongly differ from the true ones. The hypocentral errors applied correspond approximately to the limits of convergence of such methods at $10 \mathrm{~Hz}$. When using longer wavelengths and distances, the initial errors may be scaled by the same factor: for example using distances of about $500 \mathrm{~km}$ at $1 \mathrm{~Hz}$, a correct model may be retrieved starting from as far as $4 \mathrm{~km}$ from it.
Figure 2 shows how the $3 S$ method converges to the exact model in 15 iterations, while Fig. 3 shows the diverging behavior of the $2 S$ method. The best fit using the $2 S$ method is obtained at the second iteration, and is similar to the $3 S$ model at the same level, but remains poor for a synthetic case. It shows clearly how the results may be in error when inverting different classes of parameters together. The $2 S$ procedure explores afterward only local minima and no convergence is observed in 99 iterations. We used relatively small damping ratios $(0.001$ for the subsystems related to the mechanism and the source time function, 0.0001 for the location), so that the inversions are almost unconstrained and may fully depart from the initial model. When using neglectable damping (0.00001), the two methods become unstable, while when applying a stronger damping (0.01), they converge to a stable model in between the initial and the true ones, but of course not perfect. The main point is that the mechanism, the source time function and the location may diverge when two classes of parameters are linked together. While separating them, the convergence of each class may even be improved 
using a different damping factor for each class (on the basis of the condition numbers or of the iterative behavior).

To complement Figs. 2 and 3, we show in Fig. 4 the true and initial data, and the best-fitting seismograms obtained using the two methods, for all of the three stations used.

\section{Conclusions}

A method for mixed model parameter inversion, based on the separation of the parameters, is proposed. It differs from existing subspace methods in the use of damped least squares and SVD procedures. The advantage of the approach is the possibility to follow and control separately model parameter changes of each subspace by choosing appropriate damping factors which are dependent on the conditioning of the subsystem and on the a priori knowledge of the various parameters. In addition, the condition number of each subsystem being smaller than the global one, the stability of the inverted solution is improved. Although this kind of separation of model parameters is not orthogonal one, but it could be a kind of quasi-orthogonal separation if the subsystem of model parameter space is properly divided, e.g., the $3 S$ separation in this paper.

A synthetic example of simultaneous determination of source history, source geometry and hypocentral location with separation in 2 or 3 classes is discussed. When the starting model is close to the "true" one and the station distribution is good, the two approaches converge to the exact solution. However, in more critical cases, with inadequate station distribution, starting model or time resolution, the two approaches may follow different convergence paths. In such cases, only the separation of all classes of parameters may provide a reliable convergence. The separation method showed in this paper is a general separation, i.e., step by step solving the linear matrix equation; it generally has a similar computation time, but needs less memory during the inversion, compared with directly solving the original linear equation.
The method of separation of parameters may therefore offer a promising tool for the inversion of multiple classes of parameters, whose general mathematical formulation is provided here.

Acknowledgments This work is supported by Innovation Project of Chinese Academy of Sciences. The author would like to express his thanks to Dr. H. Dufumier for producing the test results and reviewers for constructive suggestions.

\section{References}

Dufumier H, Michelini A, Du Z, Bondar I, Sileny J, Mao WJ, Kravanja S, Panza GF (1997) Regional structure modeling and source inversion for the 1992 Roermond earthquake. J Seismol $1: 321-340$

Kennett BLN, Sambridge MS, Williamson PR (1988) Subspace methods for large inverse problems with multiple parameter classes. Geophys J Int 94:237-247

Mao WJ, Gubbins D (1995) Simultaneous determination of time delays and stacking weights in seismic array beamforming. Geophysics 60:491-502

Mao WJ, Stuart G (1997) Transmission-reflection tomography: application to a reverse VSP data. Geophysics 62:884-894

Mao WJ, Suhadolc P (1992) Simultaneous inversion of velocity structures and hypocentral locations: application to the Friuli seismic area NE Italy. PAGEOPH 138:267-285

Mao WJ, Panza GF, Suhadolc P (1994) Linearized waveform inversion of local and near-regional events for source mechanism and rupturing processes. Geophys J Int 116:784-798

Nformi S, Mao WJ, Gubbins D (1996) Seismic source parameters in New Zealand from broad-band data. Geophys J Int 124:289-303

Pavlis GL, Booker JR (1980) The mixed discrete-continuous inverse problem: application to the simultaneous determination of earthquake hypocenters and velocity structure. J Geophys Res 85:4801-4810

Spencer C (1985) The use of partitioned matrices in geophysical inversion problems. Geophys J R Astr Soc 80:619-629

Spencer C, Gubbins D (1980) Travel-time inversion for simultaneous earthquake location and velocity structure determination in laterally varying media. Geophys J R Astr Soc 63:95-116

Tarantola A (1987) Inverse problem theory, methods for data fitting and model parameter estimation. Elsevier Ed, Amsterdam 